\title{
Research Paper: Short-term Effects of Elastic Therapeutic Taping on Static and Dynamic Balance in Chronic Stroke
}

\author{
Roghayeh Mohammadi $^{1^{*}}$ (D), Mohaddeseh Hafez Yosephi ${ }^{1}$ (D), Roya Khanmohammadi² (D), Namrata Grampurohit ${ }^{3}$ (i)
}

1. Neuromuscular Rehabilitation Research Center, Semnan University of Medical Sciences, Semnan, Iran.

2. Department of Physical Therapy, School of Rehabilitation, Tehran University of Medical Sciences, Tehran, Iran.

3. Department of Occupational Therapy, School of Rehabilitation Sciences, Thomas Jefferson University, Philadelphia, USA.

\begin{tabular}{|c|c|}
\hline $\begin{array}{l}\text { Use your device to scan } \\
\text { and read the article online }\end{array}$ & \\
\hline arping: & $\begin{array}{l}\text { Therapeutic Taping on Static and Dynamic Balance in Chronic Stroke. Basic and Clinical Neuroscience, 12(4), 541-550. http:// } \\
\text { dx.doi.org/10.32598/bcn.12.4.2979.1 }\end{array}$ \\
\hline 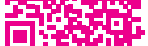 & dol'http://dx.doi.org/10.32598/bcn.12.4.2979.1 \\
\hline
\end{tabular}

\section{(c) (1) (\$)}

Article info:

Received: 20 Oct 2020

First Revision: 28 Feb 2021

Accepted: 30 Jun2021

Available Online: 01 Jul 2021

Keywords:

Stroke, Ankle, Balance, Elastic

therapeutic taping

\section{A B S T RA C T}

Introduction: The current pilot study aimed to examine the short-term effects of ankle Elastic Therapeutic Taping (ETT) on static and dynamic balance.

Methods: Twenty-Four individuals with chronic stroke were assigned to an experimental or control group ( $\mathrm{n}=12 /$ group); they both received Conventional Physical Therapy (CT) for 3 weeks, 3 times per week. The experimental group additionally underwent taping to the ankle of the paretic side continuously for 3 weeks. Standardized measures for static and dynamic balance were administered at pre-test and post-test and analyzed using Wilcoxon and Analysis of Covariance (ANCOVA).

Results: The experimental group significantly improved on two measures, Biodex anteriorposterior static $(\mathrm{P}=0.03)$ and medial-lateral dynamic $(\mathrm{P}=0.04)$ balance indices, compared to the controls. Both groups improved within their respective groups for Berg Balance Scale and Functional Reach $(\mathrm{P}<0.05)$. Static balance consistently improved across measures with the experimental intervention with large effect sizes.

Conclusion: Ankle ETT, combined with CT, may be effective in the short-term for improving static and dynamic balance in stroke, compared to CT alone. A future larger randomized trial with longer follow-up is required to establish this method's effectiveness.

\section{* Corresponding Author:}




\section{Highlights}

- Ankle taping for stroke improved Biodex measures of static and dynamic balance.

- Effect of ankle taping was largest on static balance in individuals with stroke.

- Berg Balance Scale and Functional Reach improved in both groups.

\section{Plain Language Summary}

This study includes people who experience ankle weakness due to a stroke six months before the survey. Ankle weakness can cause problems with balance on stable and unstable surfaces. The purpose of the study was to see if using an elastic, sticky tape at the ankle can improve balance for people with stroke on both stable and unstable surfaces. We asked 12 people with stroke to try out the video at their ankles for three weeks continuously while getting physical therapy three times a week. We compared their balance with 12 other people who received physical therapy but did not get the tape. We measured proportion on stable and unstable surfaces with Biodex - a machine for testing balance, and two standard clinic tests. We found that the taping group improved in the Biodex regular surface test in the frontand-back direction and Biodex unstable surface test in the side-to-side direction, compared to the group without taping. A large effect size was seen for balance on stable surfaces with taping. Both groups improved in the standard clinical tests of balance. The results can guide physical therapy for people with stroke. The study supports taping the ankle for a short duration of 3 weeks combined with physical therapy over physical therapy alone. A future study is needed to confirm these findings with a larger number of people that are randomly assigned to the two groups.

\section{Introduction}

troke causes severe disability in daily life and increases dependency (Adamson, Beswick, \& Ebrahim, 2004). Balance disorders are common in stroke and highly predictable of functional problems, disability, and fall risk (Tyson, Hanley, Chillala, Selley, \& Tallis, 2006). The incidence of falls (as high as 50\%) is reported in individuals with chronic stroke living in the community (Hyndman, Ashburn, \& Stack, 2002). Large-Scale studies reported at least one fall within two years following a stroke in $23.5 \%$ of the study participants, two or more falls in $14.2 \%$ of the participants, and a fall-related injury, like fracture in $5.4 \%$ of the participants (Callaly et al., 2015). The recovery of balance is often the central goal of physical therapy and rehabilitation for stroke survivors (Ouellette et al., 2004). Physical therapy treatment with exercise and task-specific gait training is frequently supplemented by adjunct modalities, like taping (Medina-Rincón, Bagur-Calafat, Pérez, Barrios-Franquesa, \& Girabent-Farrés, 2019). Taping with an elastic tape has gained popularity in clinical practice for its athletic appeal, non-invasive application, immediate effect, and continued use beyond the clinic. Kinesio Tape is an elastic therapeutic tape introduced by Kenzo Kase in 1996 and widely adopted for musculoskeletal conditions (Briem et al., 2011). Elastic Therapeutic Taping (ETT) application is a promising intervention that has the potential to improve proprioception, correct joint position, and stimulate or inhibit the recruitment of muscles (Jaraczewska \& Long, 2006). Particularly desirable is the ability of ETT application to train the proprioceptive system outside the clinic for an extended duration of time. Other advantages of the ETT include low cost and easy application (Kim, Choi, Lee, \& Park, 2014).

Kilbreath, Perkins, Crosbie, and McConnell (2006) recommended gluteal taping for its immediate effect on improving hip extension during the stance phase in stroke. Maguire, Sieben, Frank, and Romkes (2010) demonstrated the effects of hip abductor taping in improving hip abductor activity. These results supported the effects of taping on improved muscle activation. The improvement in muscle activation of lower extremities may contribute to stable and independent walking (Chvatal \& Ting, 2013). Studies indicated that ankle Mulligan taping significantly increased dynamic stability and gait among individuals with stroke (Hyun, Cho, \& Lim, 2015). An additional benefit is in the correction of talus equinus, i.e., studied for its usefulness in the postural control of patients with stroke; particularly, applying taping in the mediolateral direction of the ankle joint to significantly improve forward reach (Forward Reach test), balance (Berg Balance Scale), and medial-lateral displacement of the center of pressure (Rojhani-Shirazi, Amirian, \& Meftahi, 2015). Research also indicated that 
implementing ankle eversion taping improves the center of pressure sway instantly; it includes sway length and sway speed in static balance and support level in dynamic balance (Kang, Choi, \& Oh, 2013). These studies used either nonelastic taping or taped joints other than the ankle. Moreover, the current study fills this critical gap in literature.

A common problem in stroke is dropping a foot or the inability to dorsiflex the foot due to the spasticity of plantarflexors, the weakness of dorsiflexors, or sensory impairments. ETT was used to address this common impairment and thereby improve balance (Hu et al., 2019; Yaghoobi, Mahmoudi, Gholami, \& Mohammadi, 2019). Koseoglu, Dogan, Tatli, Sezgin Ozcan, and Polat (2017) investigated the effects of ETT application to the tibialis anterior on clinical variables and health-related quality of life in stroke. Accordingly, they suggested that the use of ETT as an adjunct to conventional therapies is promising for its use as an ankle training method that integrates sensory and motor aspects. Prior studies also focused on the short-term (Rojhani-Shirazi, et al., 2015; Shin, Kim, \& Kim, 2017; Yazici et al., 2015) and immediate effects of ETT application (Kim et al., 2012). A systematic review (Hu et al., 2019) highlighted multiple joints taped during ETT interventions for balance. Taping of the ankle alone has not been studied for its comparative effectiveness in stroke to improve balance when added to conventional therapy and compared to conventional therapy alone. One study reported that ankle proprioception is affected by ETT and can be a useful addition to therapy (Mohammadi, Abdollahi Khorasgani, Tabatabaei, \& Grampurohit, 2019). Furthermore, a detailed examination of the effects of ETT on static and dynamic balance is necessary to guide clinical practice. Therefore, the current study intended to study the effects of a 3-week ETT intervention when added to conventional therapy on static and dynamic balance.

\section{Methods}

The study participants included community-dwelling stroke survivors who met the inclusion criteria of chronic ( $>6$ months) stroke, good cognition (evaluated by Clock drawing test), as well as the ability to walk with or without a walking aid. The exclusion criteria consisted of aphasia, incontinence, any skin sensitivity, a history of eczema or rash with tape on the skin, and a history of falls with fractures. University Ethical Committee approved the study protocol. Besides, all research participants provided a written informed consent form before entering the study.
A clinical trial design was used in this two-group quasi-experimental controlled pilot study. The study subjects were informed of all the research procedures and demographic information obtained before testing. To describe the sample, the following assessments were administered: Fugl-Meyer Assessment (FMA) for paretic Lower Extremity (LE) function and the modified Ashworth scale for spasticity for paretic plantarflexor muscles in the study groups. The FMA-LE cut-off score of $\geq 21$ was considered high mobility function (Kwong $\& \mathrm{Ng}, 2019)$. Randomization was not performed due to the pilot nature of the study and the risk of contamination (Torgerson, 2001); the study participants frequently interacted with each other within the clinic setting, and the tape being on a visible body part was limited the concealment of allocation.

The primary outcome of overall static and dynamic stability was measured using the Biodex Balance System (Biodex Medical Systems, Inc., Shirley, NY, USA). It is a valid and reliable device for assessing postural stability on both stable and unstable surfaces (Schmitz \& Arnold, 1998). The multidirectional postural sway of the participant during standing on static and dynamic platforms was measured by Biodex. It has a circular platform that moves freely in the Anterior/Posterior (AP) and Medial/ Lateral (ML) directions. The platform remained stable (without tilting) in the static postural stability task. The difficulty of the dynamic postural stability task can vary from stability level 12 (minimum tilt, maximum stability) to stability level 1 (maximum tilt, minimum stability). The results of pre-testing of the Biodex for stroke subjects by this research team suggested that the study participants were unable to complete the dynamic tests of stability level $<9$ for $30 \mathrm{sec}$. Therefore, the level of 10 , as the lowest instability level were used in the study. The study participants were instructed to stand on the platform, without touching the handrails during the test, while they were instructed to hold the handrail if they lost balance. They stood barefoot with their arms near the body. All assessments were conducted with eyes open for 30 secs (Schmitz \& Arnold, 1998). The data of postural stability in different directions was obtained by Biodex after completing each test. The A/P, M/L, and overall indices represent platform displacements in the sagittal, frontal, and a combination of both planes, respectively. A reduction in these indices indicates improvement in postural control (Schmitz \& Arnold, 1998).

To conduct the Timed Up and Go (TUG), the study participants were instructed to stand up from a chair, walk $3 \mathrm{~m}$ at a self-selected speed, turn around, and sit down. The time required to perform this task was measured 
and a lower score indicated better function. The TUG was used as a valid measure of basic mobility skills after stroke (Hafsteinsdottir, Rensink, \& Schuurmans, 2014).

The Berg Balance Scale (BBS) evaluates static balance. It involves the following items: reaching forward with stretched arm, standing with eyes closed with one foot in front, turning, retaking an object from the floor, standing on one foot, sitting to stand, turning $360^{\circ}$, standing, placing the other foot on a stool, transferring, standing with feet together, and standing to sit without support. The scale's items are scored from 0 to 4 . The maximum score is 56 . Higher BBS scores indicate better stability indices. The BBS is valid and reliable for individuals with stroke (Sahin et al., 2008).

To perform the Functional Reach (FR) test, the study subjects were requested to stand near the wall and reach forward with an outstretched arm. The maximum distance that patients could reach forward without losing balance or taking a step from a quiet stance was recorded. The FR test assesses static balance and a higher score represents better balance (Wernick-Robinson, Krebs, \& Giorgetti, 1999). All outcomes were measured before and after treatment in both stroke groups.

The examined subjects were assigned to two groups of experimental and control. The control group received Conventional Therapy (CT) and the experimental group received CT plus ankle ETT. ETT was applied to the ankle of the paretic side for 3 weeks. Taping was performed by a physiotherapist who was trained for this protocol
(Mohammadi et al., 2019). Furthermore, the study subjects were taped according to the method described by Yazici and colleagues; the detailed description and figures can be found in their full article (Yazici et al., 2015). Tape application was performed in the following steps: 1) to inhibit the gastrocnemius muscle, the tape $(5 \mathrm{~cm}$ wide) anchored at the heel and split to end at the medial and lateral heads of the gastrocnemius muscle like a "Y" strip with $0 \%$ tension on the ends and $15 \%$ tension in the middle; 2 ) to facilitate (using functional correction method) dorsiflexors, the tape anchored at the metatarsals over the dorsum of the foot and ended over the anterior aspect of the tibia with the paper-off technique to end with no tension like an "I" strip; 3) to evert the subtalar joint, providing for correct foot contact with the ground, the tape began from the medial malleolus, wrapped around the sole, and was attached above the lateral malleolus with a long tape; 4) to correct the distal fibula, the last piece of tape was wrapped around the distal leg beginning from the anterior ankle, wrapping around posteriorly and ending on the anterior ankle from the lateral side. The ankle was placed in the neutral position during taping, with the subject prone for the first step and supine for the next three steps (Yazici et al., 2015). The examined subjects were instructed to perform all daily activities they engaged in including showers, with continued use of the tape on their ankle. The tape was changed during each intervention session by the physiotherapist. CT included electrical stimulation of paretic quadriceps muscle plus balance training and exercises. First, the subjects were trained with eyes open while standing on both legs on the tilt board. Then, they progressed to one leg on the tilt

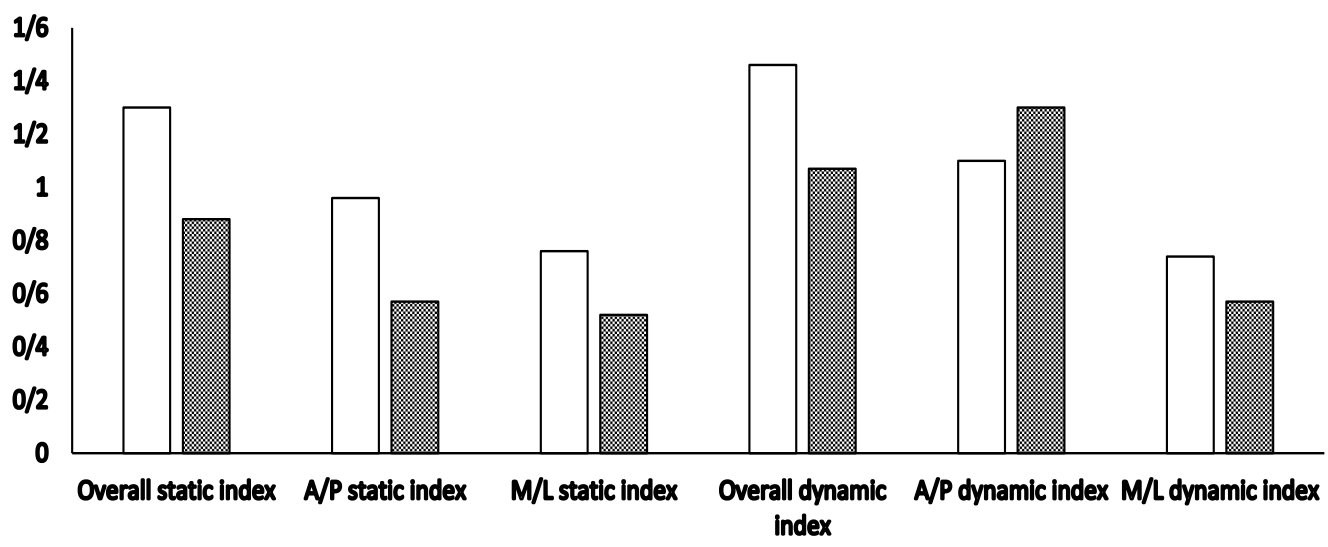

$\square$ Experimental group Pre $\quad$ Experimental group post

Figure 1. Static and dynamic stability indices, mean values before and after training for the experimental group that received conventional therapy plus elastic therapeutic taping

A/P: Antero-posterior; M/L: Medio-lateral, hollow bar for before training, full bar for after training. 
board. Parallel bars were used for safety. Both groups were trained for 3 sessions a week for 3 weeks. The experimental and control groups visited the clinic for training on even and odd days, respectively.

The obtained data were presented as Mean \pm Standard Deviation (SD) for continuous variables and frequency (\%) for categorical variables. To compare the study groups at baseline for homogeneity, the Independent Samples t-test was used for continuous variables and the Chi-squared test was applied for categorical variables. The scores between the study groups were compared using Analysis of Covariance (ANCOVA) on log-transformed data. Log-Transformation was performed due to skewness in the data and to account for non-normality due to the effects of a small sample size. None of the baseline variables were significant and did not qualify as covariates. The study did not randomize the participants between the two groups; thus, the pre-test scores for the outcome variables were used as covariates in the model. The changes from pre-test to post-test were analyzed using Wilcoxon sign ranked tests, by a conservative approach. The Cohen's d Effect Size (ES) (Cohen, 1988) and their corresponding confidence intervals for changes within and between groups were also computed to inform sample size calculations for future studies. The ESs were interpreted as: $\leq 0.2$, small; around 0.5 , medium; $\geq 0.8$, large. All the differences were considered significant at 0.05 levels, and the data analysis was performed using SPSS v. 23.

\section{Results}

A convenience sample of 24 participants was recruited. The research participants were consecutively assigned to the experimental [7F/5M; Body Mass Index (BMI): $\left.26.79 \pm 3.8 \mathrm{~kg} / \mathrm{m}^{2}\right]$, or control (7F/5M; BMI: $26.52 \pm 3.10$ $\mathrm{kg} / \mathrm{m}^{2}$ ) groups. There were no adverse events, drop-outs, or missing data in the study. The experimental group participants had a Mean \pm SD age of $66.75+3.9$ years. There were $66 \%$ experimental group participants with rightside affected and their Mean \pm SD duration of post-stroke was measured as $24+6.44$ months. The control group participants had a Mean \pm SD age of $69.67+6.34$ years. The control group had $58.3 \%$ with right-side affected and Mean \pm SD duration of post-stroke as $25.5+9.27$ months. Both groups had high mobility function greater than 21 out of 34 on the FMA-LE (Kwong \& Ng, 2019) for all participants. Spasticity in plantar flexors was $\leq 2$ on the Modified Ashworth Scale for $83.3 \%$ of all study participants. No significant difference was observed between the two groups for demographic variables before the intervention $(\mathrm{P}>0.05)$. Baseline data regarding demographic variables are presented in Table 1.

Table 2 lists the Mean \pm SD values, and change values for each variable before and after intervention in both groups along with Figures 1 and 2. The experimental group significantly performed better than the controls on Biodex A/P static $(\mathrm{P}=0.03)$ and $\mathrm{M} / \mathrm{L}$ dynamic $(\mathrm{P}=0.04)$ balance indices after controlling the pre-test scores. All

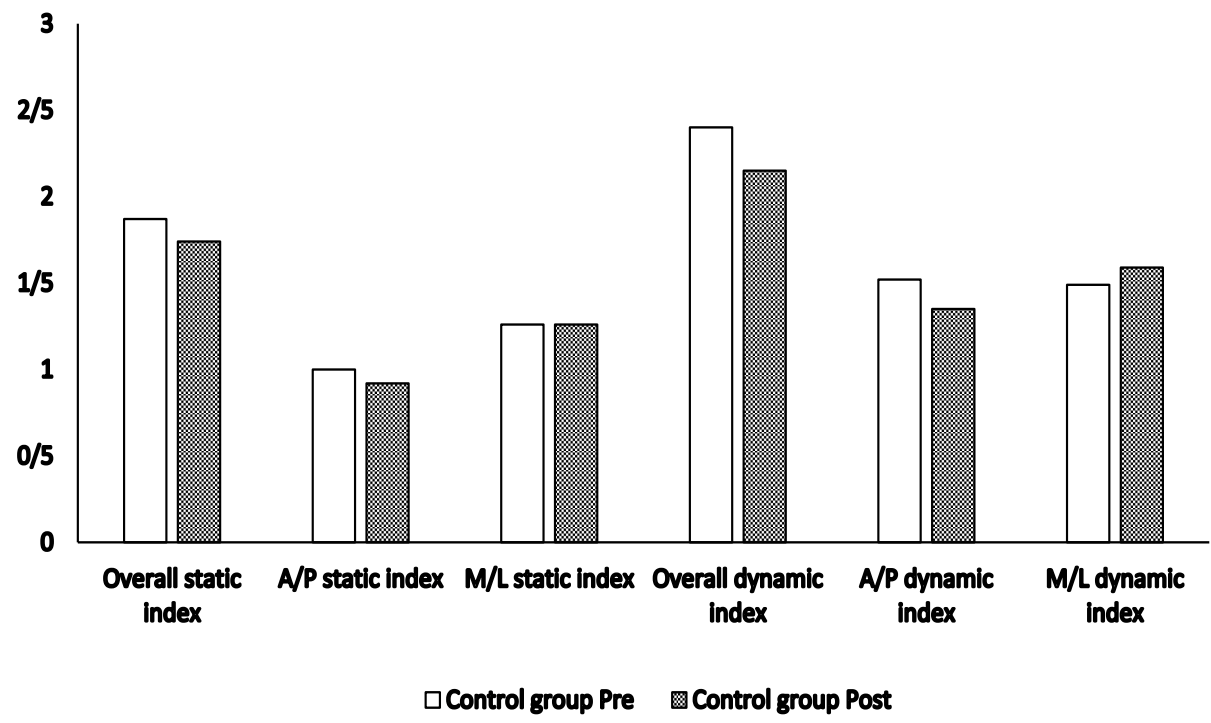

NEUR:SCIENCE

Figure 2. Static and dynamic stability indices, mean values before and after training for the control group that received conventional therapy only.

A/P: Antero-posterior; M/L: Medio-latera, hollow bar for before training, full bar for after training. 
Table 1. Characteristics of participants in the experimental and control groups at baseline

\begin{tabular}{cccc}
\hline Characteristic & Experimental group $(\mathbf{n}=\mathbf{1 2})$ & Control group $(\mathbf{n}=\mathbf{1 2})$ & P-value* \\
\hline Affected side (L/R) & $4 / 8(33.3 \% / 66.6 \%)$ & $5 / 7(41.7 \% / 58.3 \%)$ & 0.67 \\
Age (years) & $66.75 \pm 3.9$ & $69.67 \pm 6.34$ & 0.19 \\
BMI (kg/m2) & $26.79 \pm 3.8$ & $26.52 \pm 3.10$ & 0.85 \\
\hline Duration after stroke (months) & $24 \pm 6.44$ & $25.5 \pm 9.27$ & 0.65 \\
\hline FMA-LE (max=34) & $29 \pm 2.26$ & $28.41 \pm 3.02$ & 0.59 \\
Gender (M/ F) & $5 / 7(41.7 \% / 58.3 \%)$ & $5 / 7(41.7 \% / 58.3 \%)$ & 1.00 \\
Height (cm) & $168.42 \pm 9.02$ & $169.83 \pm 10.24$ & 0.72 \\
\hline MAS ankle plantarflexors & $100 \% \leq 2$ & $83.3 \% \leq 2,16.6 \%>2$ & 0.39 \\
\hline Weight (kg) & $76.42 \pm 14.1$ & $77.25 \pm 15.64$ & 0.89 \\
\hline
\end{tabular}

NEUR SCIENCE

Abbreviations: BMI, body mass index; FMA, Fugl-Meyer Assessment; F, female; L, left; LE, lower extremity; MAS, Modified Ashworth Scale; M, male; R, right. Note: Values are presented as mean + standard deviation, unless otherwise indicated. *Independent t-test used for continuous variables and chi-square test for discrete variables.

static balance measures significantly improved in the experimental group $(\mathrm{P}<0.05)$ from pre-test to post-test. Only the overall dynamic balance index significantly improved $(\mathrm{P}=0.008)$ from pre-test to post-test in the experimental group. The control group improved on the static balance measures, BBS, and FR, significantly $(\mathrm{P}<0.05)$ from pre-test to post-test. The dynamic balance measure, TUG, revealed no within- or between-group differences.

\section{Discussion}

The current study investigated the effects of ankle ETT on the static and dynamic balance measures in stroke. The collected results indicated that applying ETT along with CT improves static and dynamic balance, compared to CT alone when measured using the instrumented measure, Biodex Balance Systems. Conventional static and dynamic performance-based balance measures were unable to detect these differences. Static balance was more influenced by the experimental intervention than dynamic balance as noted in the large ES in the static balance measures.

Static balance significantly improved in all directions in the experimental group from pre-test to post-test. ETT seems to stabilize the ankle by correcting the position of the ankle; thereby improving the static indices (RojhaniShirazi et al., 2015). These findings were consistent with those of a previous study in this area (Kim et al., 2012). However, there are important differences in implement- ing the ETT between this study and Kim et al. 2012's study concerning applying the tape on the ankle; the present study corrected the fibula with the placement of the foot in eversion position. There are also differences in the duration of ETT between this study and the Kim et al. 2012's study. Kim et al. (2012) employed a longer, 8-week, protocol. The patients at the physical therapy clinic are not visited for an extended time, like 8 weeks. Besides, there was a lack of clinical utility of the Kim et al. 2012's protocol. Thus, it necessitated a shorter duration. Balance training was provided to both study groups; therefore, the performance-based static balance measures of BBS and FR revealed significant improvements within-group and ETT did not influence these measures. Previous studies indicated the positive effect of ETT application on the ankle on the BBS and FR measures (Bae, Kim, Min, \& Lee, 2015; Choi, Park, \& Lee, 2016; Kim et al., 2014; Nam, Lee, \& Cho, 2015; Rojhani-Shirazi et al., 2015); however, this was not observed in the current study. Applying ETT on the ankle can be a useful method for static balance training; accordingly, this measure reduces sway and decreases fall risk. The biomechanical explanation for this effect of ETT can be noted in the restriction of excessive joint motion and sensory effects of enhancing proprioception leading to reduced recruitment time in the ankle joint stabilizer muscles (Matsusaka, Yokoyama, Tsurusaki, Inokuchi, \& Okita, 2001). Future investigation into the maintenance of these effects is required with a long-term follow-up. 
Table 2. Comparison of balance parameters within the groups and between the groups

\begin{tabular}{|c|c|c|c|c|c|c|c|c|c|c|}
\hline \multirow{2}{*}{\multicolumn{2}{|c|}{ Variable }} & \multicolumn{4}{|c|}{ Experimental Group ( $n=12)$} & \multicolumn{3}{|c|}{ Control Group ( $n=12$ ) } & \multirow[b]{2}{*}{$\mathbf{P}$} & \multirow{2}{*}{$\frac{\text { ANCOVA }}{\mathrm{P}}$} \\
\hline & & Pre & Post & Change & $\mathbf{P}$ & Pre & Post & Change & & \\
\hline \multirow{5}{*}{ 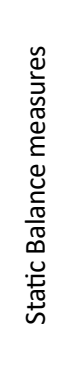 } & $\begin{array}{l}\text { Overall static } \\
\text { index }\end{array}$ & $1.3 \pm 0.85$ & $0.88 \pm 0.58$ & $-0.42 \pm 0.43$ & $0.003^{*}$ & $1.87 \pm 0.97$ & $1.74 \pm 1.13$ & $-0.13 \pm 0.37$ & 0.283 & 0.09 \\
\hline & $\begin{array}{c}\mathrm{A} / \mathrm{P} \\
\text { static index }\end{array}$ & $0.96 \pm 0.56$ & $0.57 \pm 0.36$ & $-0.38 \pm 0.33$ & $0.002^{*}$ & $1.00 \pm 0.46$ & $0.92 \pm 0.53$ & $-0.07 \pm 0.39$ & 0.797 & $0.03 * *$ \\
\hline & $\begin{array}{l}M / L \text { static } \\
\text { index }\end{array}$ & $0.76 \pm 0.52$ & $0.52 \pm 0.42$ & $-0.24 \pm 0.33$ & $0.017^{*}$ & $1.26 \pm 1.06$ & $1.26 \pm 1.17$ & $0 \pm 0.21$ & 0.877 & 0.07 \\
\hline & BBS (score) & $42.75 \pm 5.22$ & $50.58 \pm 3.37$ & $7.8 \pm 3.01$ & $0.002^{*}$ & $36.42 \pm 6.89$ & $41.75 \pm 8.46$ & $5.33 \pm 2.06$ & $0.002^{*}$ & 0.05 \\
\hline & $\mathrm{FR}(\mathrm{cm})$ & $20.42 \pm 2.71$ & $24.58 \pm 3.48$ & $4.17 \pm 1.8$ & $0.003^{*}$ & $16.50 \pm 6.23$ & $19.25 \pm 6.20$ & $2.75 \pm 1.14$ & $0.002^{*}$ & 0.16 \\
\hline \multirow{4}{*}{ 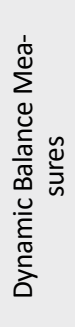 } & $\begin{array}{l}\text { Overall dy- } \\
\text { namic index }\end{array}$ & $1.46 \pm 0.32$ & $1.07 \pm 0.30$ & $-0.39 \pm 0.30$ & $0.008^{*}$ & $2.40 \pm 0.86$ & $2.15 \pm 0.92$ & $-0.25 \pm 0.43$ & 0.059 & 0.13 \\
\hline & $\begin{array}{c}\mathrm{A} / \mathrm{P} \\
\text { dynamic } \\
\text { index }\end{array}$ & $1.10 \pm 0.41$ & $1.30 \pm 1.80$ & $0.20 \pm 1.86$ & 0.153 & $1.52 \pm 0.58$ & $1.35 \pm 0.64$ & $-0.17 \pm 0.32$ & 0.097 & 0.74 \\
\hline & $\begin{array}{l}M / L \text { dynamic } \\
\text { index }\end{array}$ & $0.74 \pm 0.2$ & $0.57 \pm 0.24$ & $-0.17 \pm 0.29$ & 0.081 & $1.49 \pm 0.97$ & $1.59 \pm 1.12$ & $0.1 \pm 0.59$ & 0.621 & $0.04 * *$ \\
\hline & TUG (secs) & $13.08 \pm 2.31$ & $12.58 \pm 1.97$ & $-0.5 \pm 1.6$ & 0.323 & $20.67 \pm 20.41$ & $20.58 \pm 21.03$ & $-0.08 \pm 2.15$ & 0.822 & 0.62 \\
\hline
\end{tabular}

FR: Functional Reach; BBS: Berg Balance Scale; TUG: Timed Up and Go; A/P: Anterior/Posterior; M/L: Medial/Lateral; Values are presented as Mean \pm Standard Deviation, unless otherwise indicated. A lower score is better for all variables except BBS and FR. *Shows significance within group by Wilcoxon sign-ranked test; **Shows significance between groups by ANCOVA on log-transformed data.

Biodex $\mathrm{M} / \mathrm{L}$ dynamic index significantly increased between the study groups; the overall dynamic balance index increased in the experimental group. No other dynamic balance measures demonstrated significant changes in this study. ETT application cannot improve dynamic balance indices alone; however, Yazici et al. (2015) reported the immediate positive effect of ETT on dynamic balance, measured by the Sensory Organization Test. Similar results were reported by Shin et al. (2017). In addition to the balance training program, the proprioceptive effects of ETT could have contributed to improvements noted on dynamic balance measures; however, the effects were not consistently observed across other measures, like TUG. Future studies are recommended to investigate proprioception and its effects on balance with ETT use. There was no progression on the TUG test in the present study, i.e., contrary to the findings of Kim and associates (Kim et al., 2012). Several factors, such as strength, endurance, and speed, may influence performance on TUG and possibly ETT addition has minimal effect on this ability (Mohammadi, Ershad, Rezayinejad, Fatemi, \& Phadke, 2017). The position of ETT application, e.g., its use on quadriceps may influence functional gains on TUG (Ekiz, Aslan, \& Ozgirgin, 2015).
Two major limitations of this study were the lack of measurement of the long-term effects of ETT and the lack of randomization; both of which need to be included in future studies. The ES observed in this study will allow sample size calculation for a future larger trial that includes longer follow-up and possibly cluster randomization to address the contamination of the sample. Furthermore, further study in comparison with sham taping is suggested to reduce bias. Taping significantly improved the accuracy of sensation in those with relatively poor proprioception; although it had little effect on those with excellent proprioception (Ekiz et al., 2015). Future research with the measurement of proprioception at the ankle in stroke is recommended for ETT studies. Furthermore, the present study included individuals with chronic stroke without stratification by type of stroke and excluded individuals in the acute and subacute stages, limiting generalizability to a wider cohort of stroke survivors.

\section{Conclusion}

The present study findings demonstrated that applying ETT on the ankle, combined with CT, improves static and dynamic balance Biodex indices in individuals with chronic stroke in the short term when compared to CT 
alone. Static balance indices are high response to change with ETT application for individuals with chronic stroke. The ESs from this pilot study can inform the design of a randomized controlled trial with appropriate outcome measures, adequate power, and longer follow-up.

\section{Ethical Considerations}

\section{Compliance with ethical guidelines}

This study was approved by the Ethics Committee of the Semnan University of Medical Sciences (Code: IR.SEMUMS.REC.1395.127).

Funding

This research was supported by the research project (No.1121), Funded by the Semnan University of Medical Sciences.

\section{Authors' contributions}

Conceptualization and supervision: Roghayeh Mohammadi; Methodology: Roghayeh Mohammadi; Investigation, writing - original draft, and writing - review \& editing: Roghayeh Mohammadi, Namrata Gramprohit; Data collection: Mohaddeseh Hafez Yosephi; Data analysis: Roghayeh Mohammadi \& Roya Khanmohammadi

\section{Conflict of interest}

The authors declared no conflict of interest.

\section{Acknowledgments}

The authors wish to thank all stroke patients who kindly participated in this research; also, they thank the Neuromuscular Rehabilitation Research Center of Semnan University of Medical Sciences.

\section{References}

Adamson, J., Beswick, A., \& Ebrahim, Sh. (2004). Is stroke the most common cause of disability? Journal of Stroke and Cerebrovascular Diseases, 13(4), 171-7. [DOI:10.1016/j.jstrokecerebrovasdis.2004.06.003] [PMID]

Bae, Y. H., Kim, H. G., Min, K. S., \& Lee, S. M. (2015). Effects of lower-leg kinesiology taping on balance ability in stroke patients with foot drop. Evidence-Based Complementary and Alternative Medicine, 2015, 125629. [DOI:10.1155/2015/125629] [PMID] [PMCID]
Briem, K., Eythörsdöttir, H., Magnúsdóttir, R. G., Pálmarsson, R., Rúnarsdöttir, T., \& Sveinsson, T. (2011). Effects of kinesio tape compared with nonelastic sports tape and the untaped ankle during a sudden inversion perturbation in male athletes. Journal of Orthopaedic \& Sports Physical Therapy, 41(5), 328-35. [DOI:10.2519/jospt.2011.3501] [PMID]

Callaly, E. L., Ni Chroinin, D., Hannon, N., Sheehan, O., Marnane, M., \& Merwick, A., et al. (2015). Falls and fractures 2 years after acute stroke: The North Dublin Population Stroke Study. Age and Ageing, 44(5), 882-6. [DOI:10.1093/ageing/ afv093] [PMID]

Choi, Y. K., Park, Y. H., \& Lee, J. H. (2016). Effects of kinesio taping and Mcconnell taping on balance and walking speed of hemiplegia patients. Journal of Physical Therapy Science, 28(4), 1166-9. [DOI:10.1589/jpts.28.1166] [PMID] [PMCID]

Chvatal, S. A., \& Ting, L. H. (2013). Common muscle synergies for balance and walking. Frontiers in Computational Neuroscience, 7:48. [DOI:10.3389/fncom.2013.00048] [PMID] [PMCID]

Cohen, J. (1988). Statistical power analysis for the behavioral sciences. $2^{\text {nd }}$ Ed. Hilllsdale, NJ. Lawrence Erlbaum Associates. https:/ / books.google.com/books?id=cIJH0lR33bgC\&source =gbs_navlinks_s

Ekiz, T., Aslan, M. D., \& Özgirgin, N. (2015). Effects of kinesio tape application to quadriceps muscles on isokinetic muscle strength, gait, and functional parameters in patients with stroke. Journal of Rehabilitation Research and Development, 52(3), 323-31. [DOI:10.1682/JRRD.2014.10.0243] [PMID]

Hafsteinsdóttir, T. B., Rensink, M., \& Schuurmans, M. (2014) Clinimetric properties of the timed up and go test for patients with stroke: A systematic review. Topics in Stroke Rehabilitation, 21(3), 197-210. [DOI:10.1310/tsr2103-197] [PMID]

Hu, Y., Zhong, D., Xiao, Q., Chen, Q., Li, J., \& Jin, R. (2019). Kinesio taping for balance function after stroke: A systematic review and meta-analysis. Evidence-Based Complementary and Alternative Medicine, 2019, 8470235. [DOI:10.1155/2019/8470235] [PMID] [PMCID]

Hyndman, D., Ashburn, A., \& Stack, E. (2002). Fall events among people with stroke living in the community: Circumstances of falls and characteristics of fallers. Archives of Physical Medicine and Rehabilitation, 83(2), 165-70. [DOI:10.1053/ apmr.2002.28030] [PMID]

Hyun, K. H., Cho, H. Y., \& Lim, C. G. (2015). The effect of knee joint Mulligan taping on balance and gait in subacute stroke patients. Journal of Physical Therapy Science, 27(11), 3545-7. [DOI:10.1589/jpts.27.3545] [PMID] [PMCID]

Jaraczewska, E., \& Long, C. (2006). Kinesio ${ }^{\circledR}$ taping in stroke: Improving functional use of the upper extremity in hemiplegia Topics in Stroke Rehabilitation, 13(3), 31-42. [DOI:10.1310/33KAXYE3-QWJB-WGT6] [PMID]

Kang, M. H., Choi, S. H., \& Oh, J. S. (2013). Postural taping applied to the low back influences kinematics and EMG activity during patient transfer in physical therapists with chronic low back pain. Journal of Electromyography and Kinesiology, 23(4), 787-93. [DOI:10.1016/j.jelekin.2013.02.009] [PMID]

Kilbreath, S. L., Perkins, S., Crosbie, J., \& McConnell, J. (2006) Gluteal taping improves hip extension during stance phase of walking following stroke. Australian Journal of Physiotherapy 52(1), 53-6. [DOI:10.1016/S0004-9514(06)70062-9] 
Kim, W. I., Choi, Y. K., Lee, J. H., \& Park, Y. H. (2014). The effect of muscle facilitation using kinesio taping on walking and balance of stroke patients. Journal of Physical Therapy Science, 26(11), 1831-4. [DOI:10.1589/jpts.26.1831] [PMID] [PMCID]

Kim, Y. R., Kim, J. I., Kim, Y. Y., Kang, K. Y., Kim, B. K., \& Park, J. H., et al. (2012). Effects of ankle joint taping on postural balance control in stroke patients. International Academy of Physical Therapy Research, 3(2), 446-52. [DOI:10.5854/ JIAPTR.2012.10.30.446]

Koseoglu, B. F., Dogan, A., Tatli, H. U., Sezgin Ozcan, D., \& Polat, C. S. (2017). Can kinesio tape be used as an ankle training method in the rehabilitation of the stroke patients? Complementary Therapies in Clinical Practice, 27, 46-51. [DOI:10.1016/j. ctcp.2017.03.002] [PMID]

Kwong, P. W. H., \& Ng, S. S. M. (2019). Cutoff score of the lowerextremity motor subscale of fugl-meyer assessment in chronic stroke survivors: A cross-sectional study. Archives of Physical Medicine and Rehabilitation, 100(9), 1782-7. [DOI:10.1016/j. apmr.2019.01.027] [PMID]

Maguire, C., Sieben, J. M., Frank, M., \& Romkes, J. (2010). Hip abductor control in walking following stroke -- the immediate effect of canes, taping and TheraTogs on gait. Clinical Rehabilitation, 24(1), 37-45. [DOI:10.1177/0269215509342335] [PMID]

Matsusaka, N., Yokoyama, Sh., Tsurusaki, T., Inokuchi, Sh., \& Okita, M. (2001). Effect of ankle disk training combined with tactile stimulation to the leg and foot on functional instability of the ankle. The American Journal of Sports Medicine, 29(1), 2530. [DOI:10.1177/03635465010290010901] [PMID]

Medina-Rincón, A., Bagur-Calafat, C., Pérez, L. M., BarriosFranquesa, A. M., \& Girabent-Farrés, M. (2019). Development and validation of an exercise programme for recovery balance impairments in poststroke patients. Journal of Stroke and Cerebrovascular Diseases, 28(11), 104314. [DOI:10.1016/j.jstrokecerebrovasdis.2019.104314] [PMID]

Mohammadi, R., Abdollahi Khorasgani, M., Tabatabaei, M. S., \& Grampurohit, N. (2019). Effects of elastic therapeutic taping on joint position sense of the ankle in stroke survivors. American Journal of Physical Medicine E Rehabilitation, 98(9), 830-4. [DOI:10.1097/PHM.0000000000001191] [PMID]

Mohammadi, R., Ershad, N., Rezayinejad, M., Fatemi, E., \& Phadke, C. P. (2017). Functional effects of treadmill-based gait training at faster speeds in stroke survivors: A prospective, single-group study. International Journal of Rehabilitation Research, 40(3), 275-8. [DOI:10.1097/MRR.0000000000000231] [PMID]

Nam, C. W., Lee, J. H., \& Cho, S. H. (2015). The effect of nonelastic taping on balance and gait function in patients with stroke. Journal of Physical Therapy Science, 27(9), 2857-60. [DOI:10.1589/jpts.27.2857] [PMID] [PMCID]

Ouellette, M. M., LeBrasseur, N. K., Bean, J. F., Phillips, E., Stein, J., \& Frontera, W. R., et al. (2004). High-intensity resistance training improves muscle strength, self-reported function, and disability in long-term stroke survivors. Stroke, 35(6), 1404-9. [DOI:10.1161/01.STR.0000127785.73065.34] [PMID]

Rojhani-Shirazi, Z., Amirian, Sh., \& Meftahi, N. (2015). Effects of ankle kinesio taping on postural control in stroke patients. Journal of Stroke and Cerebrovascular Diseases, 24(11), 2565-71. [DOI:10.1016/j.jstrokecerebrovasdis.2015.07.008] [PMID]
Sahin, F., Yilmaz, F., Ozmaden, A., Kotevolu, N., Sahin, T., \& Kuran, B. (2008). Reliability and validity of the Turkish version of the Berg Balance Scale. Journal of Geriatric Physical Therapy, 31(1), 32-7. [DOI:10.1519/00139143-200831010-00006] [PMID]

Schmitz, R., \& Arnold, B. (1998). Intertester and intratester reliability of a dynamic balance protocol using the biodex stability system. Journal of Sport Rehabilitation, 7(2), 95-101. [DOI:10.1123/jsr.7.2.95]

Shin, Y. J., Kim, S. M., \& Kim, H. S. (2017). Immediate effects of ankle eversion taping on dynamic and static balance of chronic stroke patients with foot drop. Journal of Physical Therapy Science, 29(6), 1029-31. [DOI:10.1589/jpts.29.1029] [PMID] [PMCID]

Torgerson, D. J. (2001). Contamination in trials: Is cluster randomisation the answer? BMI, 322(7282), 355-7. [DOI:10.1136/ bmj.322.7282.355] [PMID] [PMCID]

Tyson, S. F., Hanley, M., Chillala, J., Selley, A., \& Tallis, R. C. (2006). Balance disability after stroke. Physical Therapy, 86(1), 30-8. [DOI:10.1093/ptj/86.1.30] [PMID]

Wernick-Robinson, M., Krebs, D. E., \& Giorgetti, M. M. (1999). Functional reach: Does it really measure dynamic balance? Archives of Physical Medicine and Rehabilitation, 80(3), 262-9. [DOI:10.1016/S0003-9993(99)90136-3]

Yaghoobi, M., Mahmoudi, Z., Gholami, M., \& Mohammadi, R. (2019). Effects of lower leg therapeutic taping on balance in central nervous system diseases: A systematic review of literature. Middle East Journal of Rehabilitation and Health Studies, 6(3), e91974. [DOI:10.5812/mejrh.91974]

Yazici, G., Guclu-Gunduz, A., Bayraktar, D., Aksoy, S., Nazliel, B., \& Kilinc, M., et al. (2015). Does correcting position and increasing sensorial input of the foot and ankle with kinesio taping improve balance in stroke patients? NeuroRehabilitation, 36(3), 345-53. [DOI:10.3233/NRE-151223] [PMID] 
This Page Intentionally Left Blank 